\title{
Synthetic Dimensions and Spin-Orbit Coupling with an Optical Clock Transition
}

\author{
L. F. Livi, ${ }^{1,5}$ G. Cappellini, ${ }^{2,5}$ M. Diem,${ }^{3,4}$ L. Franchi, ${ }^{2}$ C. Clivati,${ }^{4}$ M. Frittelli, ${ }^{4}$ F. Levi, ${ }^{4}$ \\ D. Calonico, ${ }^{4}$ J. Catani, ${ }^{5,1,6}$ M. Inguscio, ${ }^{2,1,5}$ and L. Fallani ${ }^{2,1,5,6,}$ \\ ${ }^{1}$ LENS European Laboratory for Nonlinear Spectroscopy, I-50019 Sesto Fiorentino, Italy \\ ${ }^{2}$ Department of Physics and Astronomy, University of Florence, I-50019 Sesto Fiorentino, Italy \\ ${ }^{3}$ ILP Institut für Laserphysik, Universität Hamburg, D-20355 Hamburg, Germany \\ ${ }^{4}$ INRIM Istituto Nazionale di Ricerca Metrologica, I-10135 Torino, Italy \\ ${ }^{5}$ INO-CNR Istituto Nazionale di Ottica del CNR, Sezione di Sesto Fiorentino, I-50019 Sesto Fiorentino, Italy \\ ${ }^{6}$ INFN Istituto Nazionale di Fisica Nucleare, Sezione di Firenze, I-50019 Sesto Fiorentino, Italy
}

(Received 18 September 2016; published 23 November 2016)

\begin{abstract}
We demonstrate a novel way of synthesizing spin-orbit interactions in ultracold quantum gases, based on a single-photon optical clock transition coupling two long-lived electronic states of two-electron ${ }^{173} \mathrm{Yb}$ atoms. By mapping the electronic states onto effective sites along a synthetic "electronic" dimension, we have engineered fermionic ladders with synthetic magnetic flux in an experimental configuration that has allowed us to achieve uniform fluxes on a lattice with minimal requirements and unprecedented tunability. We have detected the spin-orbit coupling with fiber-link-enhanced clock spectroscopy and directly measured the emergence of chiral edge currents, probing them as a function of the flux. These results open new directions for the investigation of topological states of matter with ultracold atomic gases.
\end{abstract}

DOI: 10.1103/PhysRevLett.117.220401

Ultracold atoms are emerging as a very versatile platform for the investigation of topological states of matter [1], thanks to the possibility of using laser light to synthesize artificial gauge fields $[2,3]$ and to engineer lattices with topological band structures [4-8]. A prime element for the emergence of nontrivial topological properties is the presence of spin-orbit coupling (SOC) [9,10], locking the spin of the particles to their motion. This interaction was first synthesized in cold atomic gases by using twophoton Raman transitions [11] coupling two hyperfine spin states with a transfer of momentum. The coupling between spin states also enables a new powerful tool for engineering topological states of matter, which relies on the "synthetic dimension" (SD) concept [12,13]. According to this approach, the internal states of an atom are treated as effective sites along a synthetic lattice dimension, and coherent coupling between them is interpreted in terms of an effective tunneling. This idea has recently been realized in Refs. [14,15], where synthetic flux ladders have been implemented by using the spin degree of freedom, and has allowed the first observation of chiral edge states in ultracold atomic systems. Its extension has inspired several proposals, opening the way, e.g., to the observation of new quantum states $[16,17]$, to the detection of fractional charge pumping $[18,19]$, or to the observation of the four-dimensional quantum Hall effect [20].

In this Letter, we demonstrate that SOC and SDs can be efficiently implemented by exploiting different degrees of freedom, specifically, the long-lived electronic state of alkaline-earth(-like) atoms. By using the technology developed in the context of optical atomic clocks, we induce a coherent coupling between the ground state $g={ }^{1} S_{0}$ and the metastable state $e={ }^{3} P_{0}$ (lifetime $\sim 20 \mathrm{~s}$ ) of ultracold ${ }^{173} \mathrm{Yb}$ atoms. Since the two states are separated by an optical energy, it is possible to have a sizable transfer of momentum with a single-photon transition, as pointed out

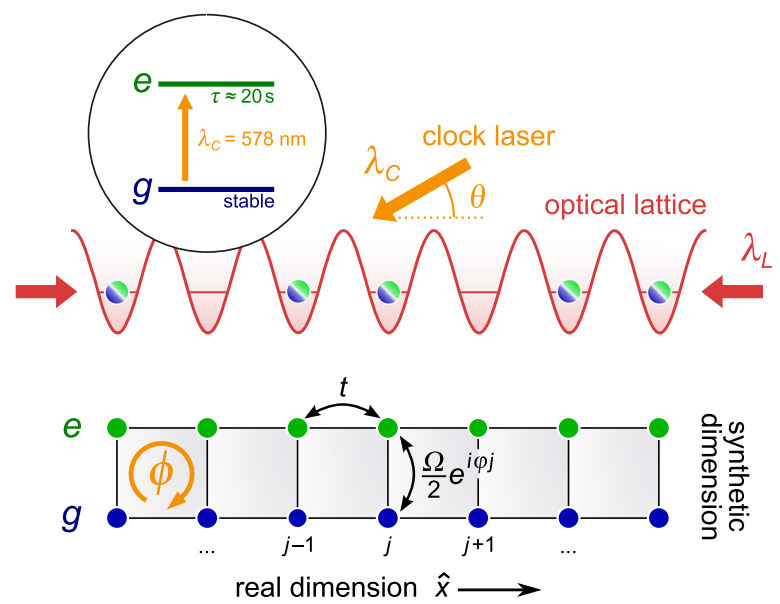

FIG. 1. Sketch of the setup. Ultracold ${ }^{173} \mathrm{Yb}$ fermions are trapped in 1D chains by an optical lattice at wavelength $\lambda_{L}$. An ultranarrow clock laser with wavelength $\lambda_{C}$ drives the singlephoton transition between the ground state $g={ }^{1} S_{0}$ and the long-lived electronic state $e={ }^{3} P_{0}$. The laser momentum transfer $\delta k=2 \pi \cos \theta / \lambda_{C}$ results in a locking between internal state (interpreted as an effective pseudospin) and atomic momentum. The electronic state can also be treated as an effective synthetic dimension made by two sites connected with a coherent tunneling, resulting in a two-leg ladder pierced by a synthetic magnetic flux per plaquette $\phi=\pi \delta k / k_{L}$, which can be tuned by adjusting the angle $\theta$. 
in a first proposal for the production of gauge fields with $\mathrm{Yb}$ atoms in optical superlattices [21]. This single-photon coupling induces a non-negligible SOC when the two states are treated as spin projection states of an effective $J=1 / 2$ spin, as proposed in Ref. [22]. Hereafter, we will use the term "spin" to refer to this pseudospin degree of freedom. An advantage of this pure two-level scheme over the Raman scheme employed so far is that it avoids the detrimental effect of near-resonant intermediate states that were observed to cause strong heating in alkali fermions $[23,24]$ hampering the observation of true many-body effects [25,26]. Moreover, the implementation of this strategy relies on a simpler configuration consisting of a single laser beam, which simplifies the alignment procedure and does not suffer from interferometric instabilities. The effectiveness of this strategy, in terms of easier experimental implementation, allows us to study chiral edge currents in synthetic fermionic ladders with a tunable synthetic magnetic flux. Although tunable gauge fields were demonstrated in different settings (e.g., for atoms in the continuum [2,3]), to our knowledge we are reporting on the first experimental investigation of atoms in a ladder or lattice geometry under the action of a largely tunable uniform flux.

In the experiment, we trap ultracold Fermi gases of ${ }^{173} \mathrm{Yb}$ in a one-dimensional (1D) optical lattice potential $V(x)=s E_{R} \cos ^{2} k_{L} x$ generated by laser light at the "magic" wavelength $\lambda_{L}=2 \pi / k_{L}=759 \mathrm{~nm}$, as sketched in Fig. 1 . The depth of the optical lattice $s$ (measured in units of $E_{R}=\hbar^{2} k_{L}^{2} / 2 m$, where $m$ is the atomic mass) is the same for both the states $g$ and $e$ and determines the tunneling energy $t$ between next-neighboring lattice sites. An additional 2D transverse lattice, not depicted in Fig. 1, freezes the atomic motion along the orthogonal directions, producing an array of 1D independent fermionic wires. In order to engineer the SOC, we drive the $g-e$ transition using $\pi$-polarized light of an ultranarrow $\lambda_{C}=578 \mathrm{~nm}$ clock laser, frequency-stabilized to an ultra low expansion glass cavity, as described in Refs. [31,32]. The short-term linewidth of the clock laser is of the order of $30 \mathrm{~Hz}$ on a $1 \mathrm{~s} \mathrm{time}$ scale, as evidenced by spectroscopic measurements. In order to cancel the residual long-term drift of the laser, we lock it to a stable optical frequency reference generated at the Italian National Institute for Metrological Research (INRiM) in Turin and delivered to our laboratory in Florence through an optical fiber link infrastructure $[33,34]$. The angle $\theta$ between the clock laser and the optical lattice (see Fig. 1) can be changed, resulting in an effective, tunable momentum transfer $\delta k=2 \pi \cos \theta / \lambda_{C}$ along the direction of the atomic chains.

In a first set of experiments, we demonstrate the capability of the optical clock transition to induce the momentum transfer required for the generation of SOC. Figure 2(a) shows a diagram with the energy spectrum of the lowest lattice band for $g$ (blue line) and $e$ (green dotted line) as a function of the lattice momentum $k$, defined in units of $k_{L}$. The lattice dispersions are the same for both the states since the lattice is operated at the magic wavelength. Because of the conservation of momentum in the atom-light interaction, the clock transition connects state $|g, k\rangle$ with state $\left|e, k+\delta k / k_{L}\right\rangle$, ensuring spin-momentum locking. By performing a standard gauge transformation, it is possible to sketch the transition as a momentum-preserving process (vertical arrow) between the $g$ band and a momentum-shifted $e$ band (green thick line), emphasizing the dependence of the transition energy on $k$. Figure 2(b) shows a spectrum of the clock transition for a band insulator of spin-polarized fermions trapped in a lattice with $s=3$, corresponding to $t=2 \pi \times 220 \mathrm{~Hz}$. The spectrum shows an enhanced response at the edges, that is related to a divergent density of states induced by the van Hove singularities of the lattice [35]. The width of the spectrum is related to the momentum transfer and to the tunneling strength, being $8 t\left|\sin \left(\pi \delta k / 2 k_{L}\right)\right|$ for a fully occupied first lattice band. The observation of this peculiar line shape is a first spectroscopic signature of SOC, as pointed out in Ref. [22]. Figure 2(c) shows a collection of spectra ranging from $s=28$ to $s=3$, illustrating the crossover between clock spectroscopy in the Lamb-Dicke regime at large $s$ (where the bands are flat) to momentum-selective excitations at small $s$. The curves in Figs. 2(b) and 2(c) are predictions of a singleparticle model in which the only adjusted parameters are a vertical offset and a vertical scaling factor. The theoretical spectra have been calculated by assuming a uniform population of the lowest band (as verified experimentally from band-mapping measurements) and convolved with the experimental spectroscopic resolution function. The latter can be approximated with a power-broadened Lorentzian line profile with a half width at half maximum of $170 \mathrm{~Hz}$ (for the data in Fig. 2), derived from a fit of the measured spectrum at $s=28$. The agreement with the calculation is quite good, evidencing the capability of the optical clock excitation to address the energy band in a momentum- and energy-selective way.

In a second set of experiments, we exploit the SOC induced by the clock laser to engineer synthetic flux ladders following the SD approach [13]. In this picture, the coherent coupling between $g$ and $e$ can be interpreted as the realization of a two-leg flux ladder geometry, with the two legs corresponding to the states $g$ and $e$, a complex tunneling along the rungs with amplitude $\Omega / 2$ ( $\Omega$ being the Rabi frequency associated with the clock excitation), and a synthetic magnetic field flux $\phi=\pi \delta k / k_{L}$ per plaquette, as sketched in Fig. 1. At the single-particle level, the system is described by the Harper-Hofstadter ladder Hamiltonian (HHLH)

$$
H=-\hbar\left(t \sum_{j, \alpha} c_{j, \alpha}^{\dagger} c_{j+1, \alpha}+\frac{\Omega}{2} \sum_{j} e^{i \phi j} c_{j, e}^{\dagger} c_{j, g}\right)+\text { H.c. },
$$

where $t$ is the tunneling amplitude between neighboring sites on the same leg and $c_{j, \alpha}^{\dagger}\left(c_{j, \alpha}\right)$ are creation 
(a)

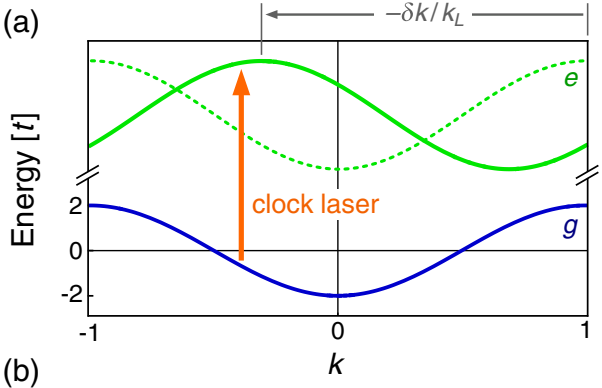

(b)

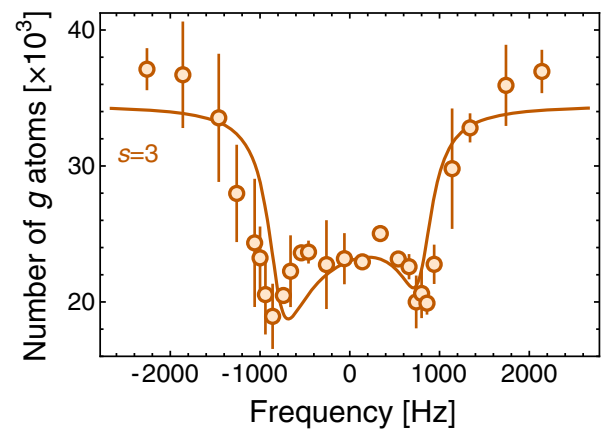

(c)

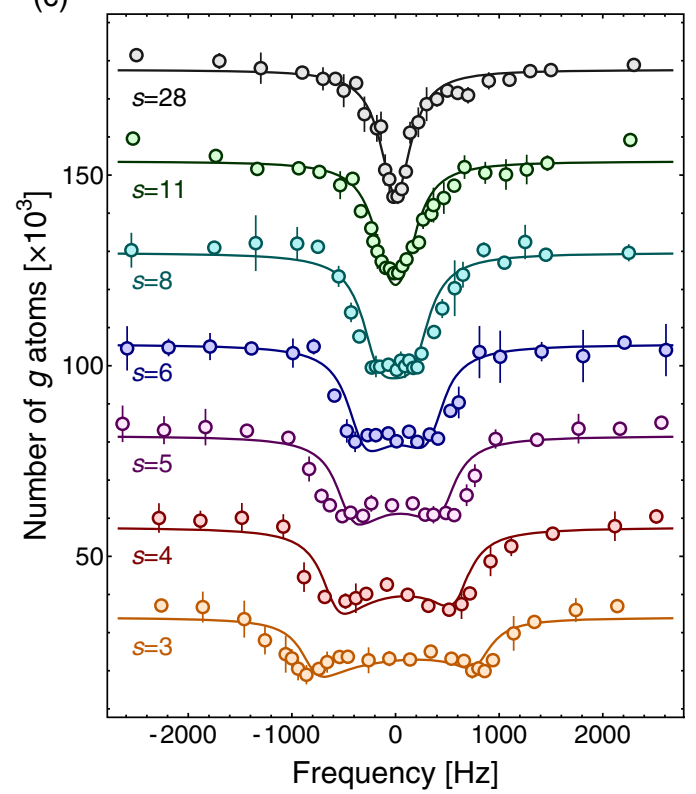

FIG. 2. Spectroscopic observation of SOC. (a) Sketch of the lattice energy bands for $g$ (blue line) and $e$ (green dashed line). Shifting the $e$ band by the SOC momentum - $\delta k$ (green solid line) allows us to represent the clock transition between the two states as a "vertical" process with a momentum-dependent energy. (b) Spectrum of the clock transition in a lattice with $s=3, t=2 \pi \times 220 \mathrm{~Hz}$ and $\delta k=1.31 k_{L}$. The plot shows the number of atoms remaining in $g$ after a 800-ms long interrogation time. The horizontal axis shows the detuning with respect to the center of the spectrum. The shape of the spectrum, featuring a broadening and an enhanced response at the edges, is a spectroscopic consequence of SOC. Points are averages over multiple measurements and error bars are standard deviations. (c) Spectra of the clock transition for different $s$. Data sets with $s \geq 4$ have been offset vertically for the sake of presentation. The curves are the result of a single-particle theoretical model (see text). All the spectra have been measured shining the clock light along the lattice direction $(\theta=0)$ and using only an additional transverse (vertical) lattice to hold the atoms against gravity.

(annihilation) operators on site $(j, \alpha)$ of the real $\hat{x}(j)$ and synthetic $(\alpha=e, g)$ dimension. Figure 3(a) shows the dressed energy bands of this Hamiltonian (after the gauge transformation described above) as a function of the lattice momentum along $\hat{x}$, with a gap opening in correspondence of the crossing between the bare-state energy curves. The color of the dressed bands indicates the $g-e$ mixing of the different momentum states, evidencing spin-momentum locking. The difference in momentum-resolved state composition between lower and higher dressed bands can be clearly seen in the images of the $g$ atoms in Fig. 3(a), obtained with the technique outlined below.

In the SD picture, spin-momentum locking corresponds to chiral edge currents traveling in opposite directions along the two legs of the ladder, as sketched in Fig. 3(a). In order to detect these currents, we prepare an equilibrium state with an adiabatic sweep of the clock laser frequency that loads the atoms, initially in $g$, into the lowest-energy (stationary) dressed state (the duration of the sweep takes $<10 \mathrm{~ms}$, i.e., 3 orders of magnitude less than the lifetime of $e$ ). Figure 3(b) shows false-color absorption images of the atoms in $g$ after a sudden switch off of the clock laser and a band-mapping procedure to measure the lattice momentum distribution [14], for $t=2 \pi \times 138 \mathrm{~Hz}$ and two different cases: without the clock laser $(\Omega=0$, left) and with the clock laser generating the flux ladder
( $\Omega=2 \pi \times 590 \mathrm{~Hz}, \phi=0.58 \pi$, right). Figure 3(c) shows the momentum distribution $n_{g}(k)$ along the lower leg of the ladder [normalized to $\int n_{g}(k) d k=1$, after integration along the transverse directions], evidencing a clear asymmetry towards positive momenta in the presence of a nonzero flux through the plaquettes. This is more evident in Fig. 3(d), where we plot the asymmetry function $h_{g}(k)=n_{g}(k)-n_{g}(-k)$. The chirality of the atomic motion can be quantified by introducing the momentum-integrated quantity $J=\int_{0}^{1} d k h_{g}(k)$, as in Ref. [14].

The single-photon excitation makes it experimentally easy to probe different fluxes $\phi$ (or, equivalently, different SOC momentum transfers $\delta k$ ), by changing the angle $\theta$ of the clock laser with respect to the orientation of the wires. In Fig. 4(a), we show the measured $J$ as a function of $\phi$. The data show a strong dependence of the chirality as a function of the flux. Noticeably, we observe an inversion of the sign of the chiral current when $\phi>\pi$. Qualitatively, this behavior can be understood on the basis of the symmetries of the system, since the flux per plaquette is defined modulo $2 \pi[J(\phi)=J(\phi+2 \pi)]$ and the current changes sign when the flux is inverted $[J(\phi)=-J(-\phi)]$ because of its chiral nature. The experimental points are compared with the result of a single-particle calculation based on the exact diagonalization of the HHLH, showing a very good agreement. The theoretical analysis includes an effective 
(a)
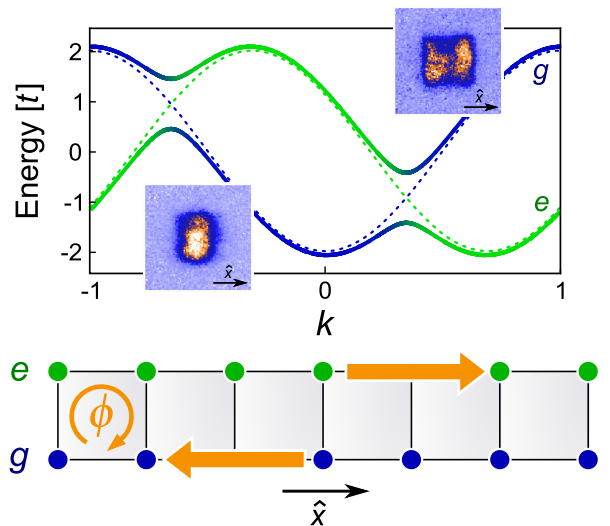

(b)

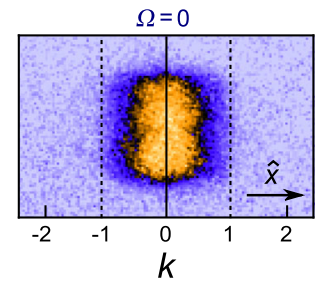

(c)

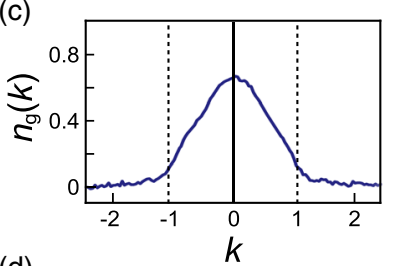

(d)
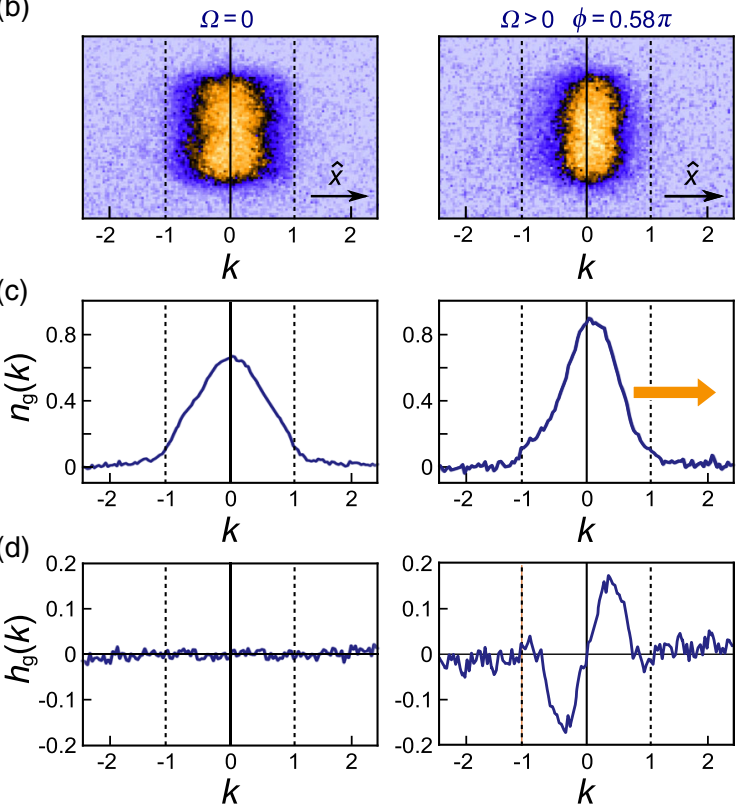

FIG. 3. Chiral currents in the synthetic ladder. (a) Sketch of the dressed (thick, solid line) and bare energy bands (thin, dashed line) for $\phi=1.31 \pi$ and $\Omega=t$. The color of the lines reflects the state composition of the dressed bands ( $e$ : green, $g$ : blue). In the SD picture, the different composition as a function of $k$ can be interpreted as chiral currents flowing in opposite directions along the two legs of the synthetic ladder. The insets show time-offlight images of the $g$ atoms for each of the two dressed bands after adiabatic loading $(\phi=1.31 \pi, t=2 \pi \times 138 \mathrm{~Hz}$, $\Omega=2 \pi \times 590 \mathrm{~Hz}$ ), evidencing complementary momentum distributions along $\hat{x}$, as expected from the state composition of the bands. (b) Time-of-flight images of the $g$ atoms without the clock laser $(\Omega=0$, left) and with the clock laser after adiabatic loading of the lowest band ( $\Omega=2 \pi \times 590 \mathrm{~Hz}, \phi=0.58 \pi$, right) for $t=2 \pi \times 138 \mathrm{~Hz}$. (c) Integrated lattice momentum distribution $n_{g}(k)$ of the atoms in the $g$ leg of the ladder for the data reported in panel (b). (d) Asymmetry function $h_{g}(k)=n_{g}(k)-n_{g}(-k)$, evidencing the chiral current induced by the nonzero flux (right).

harmonic confinement along $\hat{x}$ (as in Ref. [14]) and the average over the distribution of atomic wires (with inhomogeneous filling) realized in the experiment. We have used the temperature as an adjustable parameter, with a best-fitting value $T=0.6 \hbar t / k_{B}$. We note, however, that the
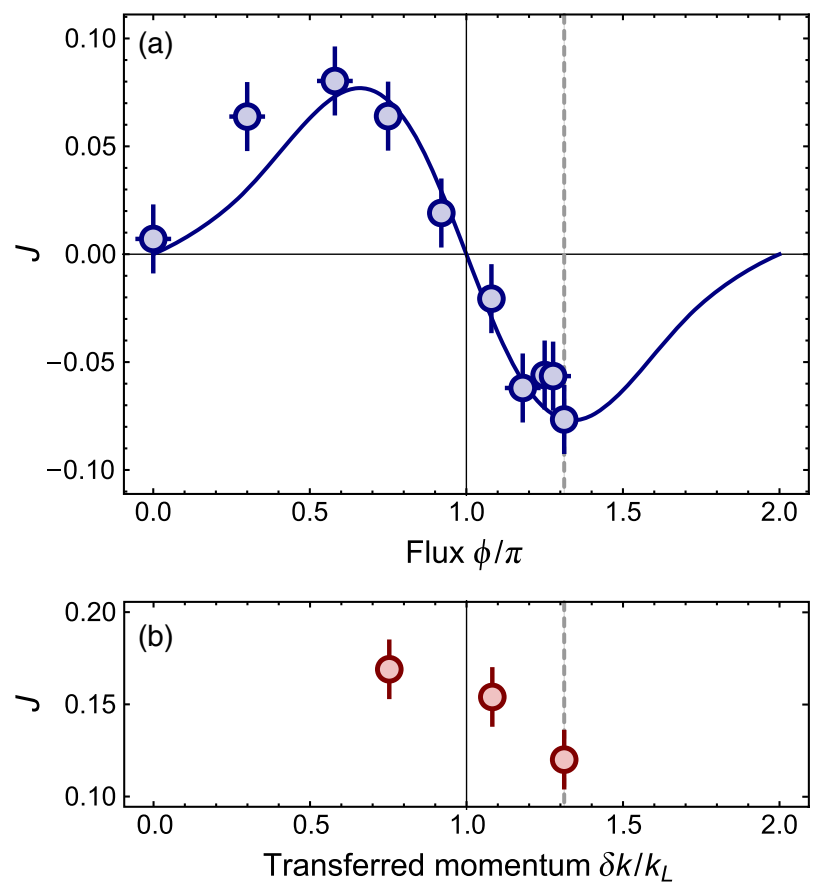

FIG. 4. (a) Dependence of the chiral current $J$ on the flux $\phi$. Vertical error bars are the standard deviation of a representative set of six measurements taken under the same conditions, while horizontal bars arise from the uncertainty on $\theta$. The dashed line marks the maximum flux that can be obtained $(\theta=0)$ with the current setup. The solid curve is the expectation of a theoretical single-particle model (see text). Experimental parameters: $t=$ $2 \pi \times 138 \mathrm{~Hz}, \Omega=2 \pi \times 590 \mathrm{~Hz}$. (b) Chiral currents in the absence of the lattice along the direction of the ladders. In this "continuum" configuration, where a $2 \mathrm{D}$ unit cell is not defined, no inversion of $J$ is observed at large SOC momentum transfer $\delta k$. We note that the horizontal scales of the two plots coincide as $\phi / \pi=\delta k / k_{L}$.

shape of the curve is robust against the fine tuning of the parameters, as it is fundamentally implied by the symmetries of the problem.

In particular, the inversion of the sign of $J$ above $\pi$ flux is a direct consequence of the discreteness of the HHLH. By removing the optical lattice along $\hat{x}$ we can synthesize a two-leg ladder in the continuum, where a unit cell is no longer defined and the symmetries arising from the discreteness of the system no longer hold. The measured values of $J$ for this configuration are reported in Fig. 4(b) as a function of the SOC momentum $\delta k$. In this case, we do not observe any inversion of $J$ when $\delta k$ exceeds $k_{L}$ [corresponding to the $\pi$ flux condition in Fig. 4(a)].

In conclusion, we have shown a new way to engineer SOC and synthetic ladders with ultracold gases of twoelectron fermions. Our system is highly promising for further studies of spin-orbit-coupled fermions in the degenerate regime. As an extension of this work, we mention the possibility of coupling different electronic and nuclear spin states with circularly polarized clock light. This would enable us to control the interactions between the two internal states by using the recently discovered ${ }^{173} \mathrm{Yb}$ orbital Feshbach 
resonance [36-38]. This would allow the investigation of synthetic flux ladders with tunable interleg interactions, and could be used to investigate SOC in atomic ${ }^{173} \mathrm{Yb}$ Fermi superfluids at the BEC-BCS crossover [39,40], e.g., to study the possible emergence of topological superfluidity. In the SD approach, we envision the possibility of combining the nuclear spin SD with the electronic SD, realizing multidimensional synthetic lattice structures with nontrivial connectivities [41,42], periodic boundary conditions, and engineered topological properties thanks to the control of the tunneling phases.

This work was funded by MIUR PRIN2012 AQUASIM, INFN FISh, and by EMPIR-15SIB05-OFTEN. We thank P. Cancio Pastor for experimental assistance and M. Mancini, G. Pagano, and C. Sias for critical reading of the manuscript. We also thank TOPTICA Photonics AG for prompt technical assistance.

Note added.-Recently, we became aware of closely related results obtained by spectroscopic measurements on ${ }^{87} \mathrm{Sr}$ nondegenerate atoms in an optical lattice clock setup [43].

${ }^{\dagger}$ Corresponding author.

fallani@lens.unifi.it

[1] N. Goldman, J. C. Budich, and P. Zoller, Nat. Phys. 12, 639 (2016).

[2] J. Dalibard, F. Gerbier, G. Juzeliūnas, and P. Öhberg, Rev. Mod. Phys. 83, 1523 (2011).

[3] N. Goldman, G. Juzeliūnas, P. Öhberg, and I. B. Spielman, Rep. Prog. Phys. 77, 126401 (2014).

[4] J. Struck, C. Ölschläger, M. Weinberg, P. Hauke, J. Simonet, A. Eckardt, M. Lewenstein, K. Sengstock, and P. Windpassinger, Phys. Rev. Lett. 108, 225304 (2012).

[5] M. Aidelsburger, M. Atala, M. Lohse, J. T. Barreiro, B. Paredes, and I. Bloch, Phys. Rev. Lett. 111, 185301 (2013).

[6] H. Miyake, G. A. Siviloglou, C. J. Kennedy, W. C. Burton, and W. Ketterle, Phys. Rev. Lett. 111, 185302 (2013).

[7] G. Jotzu, M. Messer, R. Desbuquois, M. Lebrat, T. Uehlinger, D. Greif, and T. Esslinger, Nature (London) 515, 237 (2014).

[8] L. Duca, T. Li, M. Reitter, I. Bloch, M. Schleier-Smith, and U. Schneider, Science 347, 288 (2015).

[9] V. Galitski and I. B. Spielman, Nature (London) 494, 49 (2013).

[10] H. Zhai, Rep. Prog. Phys. 78, 026001 (2015).

[11] Y.-J. Lin, K. Jiménez-García, and I. B. Spielman, Nature (London) 471, 83 (2011).

[12] O. Boada, A. Celi, J. I. Latorre, and M. Lewenstein, Phys. Rev. Lett. 108, 133001 (2012).

[13] A. Celi, P. Massignan, J. Ruseckas, N. Goldman, I. B. Spielman, G. Juzeliūnas, and M. Lewenstein, Phys. Rev. Lett. 112, 043001 (2014).

[14] M. Mancini et al., Science 349, 1510 (2015).

[15] B. K. Stuhl, H.-I. Lu, L. M. Aycock, D. Genkina, and I. B. Spielman, Science 349, 1514 (2015).

[16] S. Barbarino, L. Taddia, D. Rossini, L. Mazza, and R. Fazio, Nat. Commun. 6, 8134 (2015).
[17] J. C. Budich, C. Laflamme, F. Tschirsich, S. Montangero, and P. Zoller, Phys. Rev. B 92, 245121 (2015).

[18] T.-S. Zeng, C. Wang, and H. Zhai, Phys. Rev. Lett. 115, 095302 (2015).

[19] L. Taddia et al., arXiv:1607.07842.

[20] H. M. Price, O. Zilberberg, T. Ozawa, I. Carusotto, and N. Goldman, Phys. Rev. Lett. 115, 195303 (2015).

[21] F. Gerbier and J. Dalibard, New J. Phys. 12, 033007 (2010).

[22] M. L. Wall, A. P. Koller, S. Li, X. Zhang, N. R. Cooper, J. Ye, and A. M. Rey, Phys. Rev. Lett. 116, 035301 (2016).

[23] P. Wang, Z.-Q. Yu, Z. Fu, J. Miao, L. Huang, S. Chai, H. Zhai, and J. Zhang, Phys. Rev. Lett. 109, 095301 (2012).

[24] L. W. Cheuk, A. T. Sommer, Z. Hadzibabic, T. Yefsah, W. S. Bakr, and M. W. Zwierlein, Phys. Rev. Lett. 109, 095302 (2012).

[25] In our experimental configuration, far-off-resonant absorption towards different electronic levels yields an estimated heating rate $<10^{-7} k_{B} T_{F} / s$ (where $T_{F}$ is the Fermi temperature), completely negligible and not even detectable. This rate is about 8 orders of magnitude smaller than what was measured in experiments with Raman transitions in alkali fermions (see, e.g., Ref. [23]).

[26] Different strategies for overcoming this problem have also been recently implemented with Raman transitions in nonalkali fermions [27,28] or by implementing effective mappings using superlattice potentials [29,30].

[27] N. Q. Burdick, Y. Tang, and B. L. Lev, Phys. Rev. X 6, 031022 (2016).

[28] B. Song et al., arXiv:1608.00478.

[29] M. Atala, M. Aidelsburger, M. Lohse, J. T. Barreiro, B. Paredes, and I. Bloch, Nat. Phys. 10, 588 (2014).

[30] J. Li, W. Huang, B. Shteynas, S. Burchesky, F. Çağrı Top, E. $\mathrm{Su}$, J. Lee, A. O. Jamison, and W. Ketterle, Phys. Rev. Lett. 117, 185301 (2016).

[31] G. Cappellini, P. Lombardi, M. Mancini, G. Pagano, M. Pizzocaro, L. Fallani, and J. Catani, Rev. Sci. Instrum. 86, 073111 (2015).

[32] M. Pizzocaro, G. A. Costanzo, A. Godone, F. Levi, A. Mura, M. Zoppi, and D. Calonico, IEEE Trans. Ultrason. Ferroelectr. Freq. Control 59, 426 (2012).

[33] C. Clivati et al., Opt. Express 24, 11865 (2016).

[34] D. Calonico et al., Appl. Phys. B 117, 979 (2014).

[35] N. W. Ashcroft and D. N. Mermin, Solid State Physics (Saunders College Publishing, Fort Worth, TX, 1976).

[36] R. Zhang, Y. Cheng, H. Zhai, and P. Zhang, Phys. Rev. Lett. 115, 135301 (2015).

[37] G. Pagano, M. Mancini, G. Cappellini, L. Livi, C. Sias, J. Catani, M. Inguscio, and L. Fallani, Phys. Rev. Lett. 115, 265301 (2015).

[38] M. Höfer, L. Riegger, F. Scazza, C. Hofrichter, D. R. Fernandes, M. M. Parish, J. Levinsen, I. Bloch, and S. Fölling, Phys. Rev. Lett. 115, 265302 (2015).

[39] J. Xu, R. Zhang, Y. Cheng, P. Zhang, R. Qi, and H. Zhai, Phys. Rev. A 94, 033609 (2016).

[40] L. He, J. Wang, S.-G. Peng, X.-Ji Liu, and H. Hu, Phys. Rev. A 94, 043624 (2016).

[41] O. Boada, A. Celi, J. Rodríguez-Laguna, J. I. Latorre, and M. Lewenstein, New J. Phys. 17, 045007 (2015).

[42] E. Anisimovas et al., arXiv:1610.00709.

[43] S. Kolkowitz et al., arXiv:1608.03854. 